PROCEEDINGS OF THE

AMERICAN MATHEMATICAL SOCIETY

Volume 140, Number 3, March 2012, Pages 1041-1044

S 0002-9939(2011)11065-7

Article electronically published on July 26, 2011

\title{
AN ALMOST SCHUR THEOREM ON 4-DIMENSIONAL MANIFOLDS
}

\author{
YUXIN GE AND GUOFANG WANG
}

(Communicated by Matthew J. Gursky)

\begin{abstract}
In this short paper we prove that the almost Schur theorem, introduced by De Lellis and Topping, is true on 4-dimensional Riemannian manifolds of nonnegative scalar curvature and discuss some related problems on other dimensional manifolds.
\end{abstract}

\section{INTRODUCTION}

Very recently, De Lellis and Topping proved an interesting result about a generalization of Schur's theorem.

Theorem 1 (Almost Schur Theorem [1]). For $n \geq 3$, if $\left(M^{n}, g\right)$ is a closed Riemannian manifold with nonnegative Ricci tensor, then

$$
\int_{M}\left|R i c-\frac{\bar{R}}{n} g\right|^{2} d v(g) \leq \frac{n^{2}}{(n-2)^{2}} \int_{M}\left|R i c-\frac{R}{n} g\right|^{2} d v(g),
$$

where $\bar{R}=\operatorname{vol}(g)^{-1} \int_{M} R d v(g)$ is the average of the scalar curvature $R$ of $g$.

It is clear that the Schur theorem follows directly from Theorem 1 . The latter can be seen as a quantitative version or a stability result of the Schur Theorem. In [1] they also showed that the constant in inequality (1) is optimal and the nonnegativity of the Ricci tensor cannot be removed in general: When $n \geq 5$ they gave examples of metrics on $\mathbb{S}^{n}$ which make the ratio of the left-hand side of (11) to the right-hand side of (1) arbitrarily large. When $n=3$, they found manifolds which make the ratio arbitrary. At the end they left an open question: Inequalities of this form may hold for $n=3$ and $n=4$ with constants depending on the topology of $M$.

In this short paper we will show that Theorem 1 holds under the condition of nonnegativity of the scalar curvature for dimension $n=4$.

Theorem 2. If $n=4$ and if $\left(M^{4}, g\right)$ is a closed Riemannian manifold with nonnegative scalar curvature, then (11) holds. Moreover, equality holds if and only if $\left(M^{4}, g\right)$ is an Einstein manifold.

We first observe that inequality (1) is equivalent to

$$
\left(\int_{M} \sigma_{1}(g) d v(g)\right)^{2} \geq \frac{2 n}{n-1} \operatorname{vol}(g) \int_{M} \sigma_{2}(g) d v(g),
$$

Received by the editors April 4, 2010 and, in revised form, December 21, 2010.

2010 Mathematics Subject Classification. Primary 53C21; Secondary 58J05, 35J60.

The second-named author is partly supported by SFB/TR71 of DFG.

(C)2011 American Mathematical Society Reverts to public domain 28 years from publication 
where $\sigma_{k}(g)$ is the $k$-scalar curvature of metric $g$. Its definition will be recalled in Section 2 . Then we prove this inequality for $n=4$ by using an argument given by Gursky [3].

\section{Proof of Theorem 2}

Let us first recall the definition of the $k$-scalar curvature, which was first introduced by Viaclovsky [4 and has been intensively studied by many mathematicians. Let

$$
S_{g}=\frac{1}{n-2}\left(R i c_{g}-\frac{R_{g}}{2(n-1)} \cdot g\right)
$$

be the Schouten tensor of $g$. For an integer $k$ with $1 \leq k \leq n$ let $\sigma_{k}$ be the $k$-th elementary symmetric function in $\mathbb{R}^{n}$. The $k$-scalar curvature is

$$
\sigma_{k}(g):=\sigma_{k}\left(\Lambda_{g}\right),
$$

where $\Lambda_{g}$ is the set of eigenvalues of the matrix $g^{-1} \cdot S_{g}$. In particular, $\sigma_{1}(g)=\operatorname{tr} S$ and $\sigma_{2}=\frac{1}{2}\left((\operatorname{tr} S)^{2}-|S|^{2}\right)$. It is trivial to see that

$$
\begin{aligned}
\sigma_{1}(g) & =\frac{R}{2(n-1)}, \\
\sigma_{2}(g) & =\frac{1}{2(n-2)^{2}}\left\{-|R i c|^{2}+\frac{n}{4(n-1)} R^{2}\right\}, \\
\left|R i c-\frac{R}{n} g\right|^{2} & =|R i c|^{2}-\frac{R^{2}}{n} .
\end{aligned}
$$

From the above it is easy to have the following observation.

Observation 1. Inequality (1) is equivalent to (2).

Hence, instead of proving Theorem 2 we actually prove

Theorem 3. If $n=4$ and if $\left(M^{n}, g\right)$ is a closed Riemannian manifold with nonnegative scalar curvature, then (2) holds. Moreover, equality holds if and only if $(M, g)$ is an Einstein metric.

The proof of Theorem 3 follows closely a nice argument of Gursky [3].

Lemma 1. For any $n \geq 3$ and any closed Riemannian manifold $\left(M^{n}, g\right)$, there exists a conformal metric $g_{1} \in[g]$ satisfying

$$
\frac{2 n}{n-1} \frac{\int_{M} \sigma_{2}\left(g_{1}\right) d v\left(g_{1}\right)}{\left(\operatorname{vol}\left(g_{1}\right)\right)^{\frac{n-4}{n}}} \leq Y_{1}([g])^{2},
$$

where $Y_{1}([g])$ is the first Yamabe invariant defined by

$$
Y_{1}([g]):=\inf _{g \in[g]} \frac{\int_{M} \sigma_{1}(g) d v(g)}{(\operatorname{vol}(g))^{\frac{n-2}{n}}}
$$

and $[g]$ is the conformal class of the metric to $g$.

Here our definition of the Yamabe constant is different from the standard one by a multiple factor $\frac{1}{2(n-1)}$. 
Proof of Lemma 1. The proof follows closely an argument given by Gursky in [3]. Let $g_{1}$ be a solution of the Yamabe problem. Thus the scalar curvature, and hence $\sigma_{1}(g)$, is constant. We have a simple fact: for any $n \times n$ symmetric matrix $A$ such that

$$
\left(\sigma_{1}(A)\right)^{2} \geq \frac{2 n}{n-1} \sigma_{2}(A)
$$

equality holds if and only if the matrix is a multiple of the identity matrix. Now the following calculations lead to

$$
\frac{2 n}{n-1} \operatorname{vol}\left(g_{1}\right) \int_{M} \sigma_{2}\left(g_{1}\right) d v\left(g_{1}\right) \leq \operatorname{vol}\left(g_{1}\right) \int_{M}\left(\sigma_{1}\left(g_{1}\right)\right)^{2} d v\left(g_{1}\right)=\left(\int_{M} \sigma_{1}\left(g_{1}\right) d v\left(g_{1}\right)\right)^{2} .
$$

Here we have used the fact that $\sigma_{1}\left(g_{1}\right)$ is a constant. Therefore,

$$
\frac{2 n}{n-1} \frac{\int_{M} \sigma_{2}\left(g_{1}\right) d v\left(g_{1}\right)}{\left(\operatorname{vol}\left(g_{1}\right)\right)^{\frac{n-4}{n}}} \leq\left(\frac{\int_{M} \sigma_{1}\left(g_{1}\right) d v\left(g_{1}\right)}{\operatorname{vol}\left(g_{1}\right)^{\frac{n-2}{n}}}\right)^{2}=Y_{1}([g])^{2},
$$

since $g_{1}$ is a Yamabe solution.

Proof of Theorem 3. In the case of dimension $n=4$, it is well known that $\int_{M} \sigma_{2}(g) d v(g)$ is constant in any given conformal class. Hence by Lemma 1 we have

$$
\begin{aligned}
\frac{2 n}{n-1} \int_{M} \sigma_{2}(g) d v(g) & =\frac{2 n}{n-1} \int_{M} \sigma_{2}\left(g_{1}\right) d v\left(g_{1}\right) \leq Y_{1}([g])^{2} \\
& \leq\left(\frac{\int_{M} \sigma_{1}(g) d v(g)}{\operatorname{vol}(g)^{\frac{1}{2}}}\right)^{2} .
\end{aligned}
$$

In the last inequality we have used the condition $\sigma_{1}(g) \geq 0$, which implies that $Y_{1}([g]) \geq 0$. The equality holds if and only if the Schouten tenser $S_{g}$ is proportional to the metric $g$; i.e., $g$ is an Einstein metric.

We conjecture that Theorem 2 is true for $n=3$. To attack this conjecture one needs to study a corresponding Yamabe-type problem. The methods developed, especially in [2, for a $\sigma_{k}$-Yamabe problem would be helpful to study this problem.

\section{Note added in proof.}

1. The conjecture proposed at the end of the paper was proved in [6].

2. The rigidity of Theorem 1, i.e., equality in (1) implies that the metric is Einstein, was proved in [5], among other generalizations of Theorems 1 and 2, It was also proved independently in the last version of $[1]$.

\section{REFERENCES}

[1] C. De Lellis and P. Topping, Almost Schur Theorem, to appear in Calc. Var. PDE, arXiv 1003.3527 .

[2] Y. Ge, C.-S. Lin and G. Wang, On $\sigma_{2}$-scalar curvature, J. Diff. Geo., 84 (2010), 45-86. MR2629509

[3] M. Gursky, The principal eigenvalue of a conformally invariant differential operator, with an application to semilinear elliptic PDE, Comm. Math. Phys., 207 (1999), 131-143. MR.1724863 (2000k:58029) 
[4] J. Viaclovsky, Conformal geometry, contact geometry, and the calculus of variations, Duke Math. J., 101 (2000), 283-316. MR1738176 (2001b:53038)

[5] Y. Ge, G. Wang and C. Xia, On problems related to an inequality of DeLellis and Topping, preprint, 2011.

[6] Y. Ge and G. Wang, A new conformal invariant on 3-dimensional manifolds and its applications, arXiv 1103.3838.

Laboratoire d'Analyse et de Mathématiques Appliquées, CNRS UMR 8050, Département de Mathématiques, Université Paris Est-Créteil Val de Marne, 61 avenue du Général de Gaulle, 94010 Créteil Cedex, France

E-mail address: ge@univ-paris12.fr

Albert-Ludwigs-Universität Freiburg, Mathematisches Institut, Eckerstrasse 1, D-79104 Freiburg, Germany

E-mail address: guofang.wang@math.uni-freiburg.de 